\title{
Dynamic Gravity Cancellation in Robots with Flexible Transmissions
}

\author{
Alessandro De Luca
}

\begin{abstract}
We consider the problem of perfect cancellation of gravity effects in the dynamics of robot manipulators having flexible transmissions at the joints. Based on the feedback equivalence principle, we aim at designing feedback control laws that let the system outputs behave as those of a desired model where gravity is absent. The cases of constant stiffness (elastic joints), nonlinear flexible, and variable nonlinear flexible transmissions with antagonistic actuation are analyzed. In all these situations, viable solutions are obtained either in closed algebraic form or by a simple numerical technique. The compensated system can then be controlled without taking into account the gravity bias, which is particularly relevant for safe physical human-robot interaction tasks where such compliant manipulators are commonly used. Simulation results are reported illustrating the obtained performance.
\end{abstract}

\section{INTRODUCTION}

Robots in physical interaction with humans are conveniently controlled so as to achieve zero-gravity operation [1]. This avoids biasing the robot reaction to unintended collisions along the gradient of the gravitational potential, with a uniform and more predictable (thus safer) robot behavior in its whole workspace [2]. Perfect cancellation of gravity is trivial for fully rigid manipulators. In fact, for their standard dynamic model

$$
\boldsymbol{M}(\boldsymbol{q}) \ddot{\boldsymbol{q}}+\boldsymbol{c}(\boldsymbol{q}, \dot{\boldsymbol{q}})+\boldsymbol{g}(\boldsymbol{q})=\boldsymbol{\tau},
$$

the choice

$$
\boldsymbol{\tau}=\boldsymbol{\tau}_{g}+\boldsymbol{\tau}_{0}, \quad \boldsymbol{\tau}_{g}=\boldsymbol{g}(\boldsymbol{q})
$$

removes gravity from the picture in a complete way (i.e., both statically and dynamically), thanks to the colocation of gravity and input torques (and to the full actuation of the system). The additional command $\tau_{0}$ is left to the control designer for performing desired tasks, e.g., set-point regulation, trajectory tracking, or reaction to a contact with the environment.

However, robots intended for physical Human-Robot Interaction (pHRI) include compliant elements in their mechanical construction, in order to reduce the possibility of injuries due to unexpected collisions [3]. Robot links are designed as lightweight but rigid, while compliance is typically concentrated in the transmissions at the joints, either with finite constant stiffness $\boldsymbol{K}$, e.g., when using harmonic drives [4], or with variable (and independently actuated) nonlinear stiffness [5].

The authors are with the Dipartimento di Informatica e Sistemistica, Università di Roma "La Sapienza", Via Ariosto 25, 00185 Rome, Italy \{deluca,fflacco\}@dis.uniroma1.it

\author{
Fabrizio Flacco
}

The common dynamic model of robots with (constant) joint elasticity takes the form [6]

$$
\begin{aligned}
\boldsymbol{M}(\boldsymbol{q}) \ddot{\boldsymbol{q}}+\boldsymbol{c}(\boldsymbol{q}, \dot{\boldsymbol{q}})+\boldsymbol{g}(\boldsymbol{q})+\boldsymbol{K}(\boldsymbol{q}-\boldsymbol{\theta}) & =\mathbf{0} \\
\boldsymbol{B} \ddot{\boldsymbol{\theta}}+\boldsymbol{K}(\boldsymbol{\theta}-\boldsymbol{q}) & =\boldsymbol{\tau},
\end{aligned}
$$

where actuation torques $\tau$ appear on the motor side of the elastic joints (i.e., performing work on $\boldsymbol{\theta}$ ), while gravity loading $\boldsymbol{g}(\boldsymbol{q})$ affects primarily the dynamic behavior of the variables on the link side (i.e., $\boldsymbol{q}$ ). This non-colocation is a major problem for control. Gravity compensation laws have been proposed for the case of regulation tasks, when the link position $\boldsymbol{q}$ has to be asympotically stabilized to a desired constant value $\boldsymbol{q}_{d}$. A first solution is based on motor PD feedback with constant gravity compensation at steady state [7]

$$
\boldsymbol{\tau}_{0}=\boldsymbol{K}_{P}\left(\boldsymbol{\theta}_{d}-\boldsymbol{\theta}\right)-\boldsymbol{K}_{D} \dot{\boldsymbol{\theta}}, \quad \boldsymbol{\tau}_{g}=\boldsymbol{g}\left(\boldsymbol{q}_{d}\right),
$$

with $\boldsymbol{\theta}_{d}=\boldsymbol{q}_{d}+\boldsymbol{K}^{-1} \boldsymbol{g}\left(\boldsymbol{q}_{d}\right), \boldsymbol{K}_{P}>0$, and $\boldsymbol{K}_{D}>0$. In order to show asympotic stability by Lyapunov arguments, $\boldsymbol{K}_{P}$ should be larger than a positive parameter that bounds the norm of the gradient of $\boldsymbol{g}(\boldsymbol{q})$. Indeed, this compensation cancels gravity only in the final static condition. Since the gravity term changes with the robot configuration, an on-line compensation has been proposed in [8] by evaluating $\boldsymbol{g}$ in $\tau_{g}$ with a gravity-biased measure of the motor position

$$
\boldsymbol{\tau}_{g}=\boldsymbol{g}(\tilde{\boldsymbol{\theta}}), \quad \tilde{\boldsymbol{\theta}}=\boldsymbol{\theta}-\boldsymbol{K}^{-1} \boldsymbol{g}\left(\boldsymbol{q}_{d}\right) .
$$

While the transient performance is largely improved, the theoretical restriction on $\boldsymbol{K}_{P}$ could not be removed in the Lyapunov analysis. A better result is achieved in [9], with a gravity compensation of the form

$$
\boldsymbol{\tau}_{g}=\boldsymbol{g}(\overline{\boldsymbol{q}}(\boldsymbol{\theta}))
$$

where, for any measured motor position $\boldsymbol{\theta}, \overline{\boldsymbol{q}}(\boldsymbol{\theta})$ is computed by numerically solving $\boldsymbol{g}(\boldsymbol{q})+\boldsymbol{K}(\boldsymbol{q}-\boldsymbol{\theta})=\mathbf{0}$ (quasistatic relation). This variant relaxes the lower bound on $\boldsymbol{K}_{P}$ and asymptotic stability can be shown through a modified Lyapunov function.

All the above control laws have the merit of using only feedback from the motor variables $\boldsymbol{\theta}$ and $\dot{\boldsymbol{\theta}}$. However, none of them is able to remove completely the effects of gravity, especially in highly dynamic tasks: only a partial compensation, and not a cancellation, of the gravitational load acting on the robot link motion is obtained. In the context of robot reaction to collisions, we also note that a practical solution for compensating gravity in elastic joint robots has been proposed in [10], based on the availability 
of joint torque sensors. The use of this additional sensor can be interpreted as involving also the link position $\boldsymbol{q}$ in the control law. Furthermore, under the assumption that full state is available, it is known that all robots with elastic joints can be exactly linearized by means of a static [11] or dynamic state feedback (the latter is needed when some extra inertial terms are included in the model) [12]. This structural control property will be further exploited in this paper.

The most recent research in pHRI calls for the use of variable stiffness actuation (VSA), in which each joint is driven by two independent actuators allowing to control the link motion as well as the device stiffness [13]-[16], and to shape the compliant interaction with the environment. Actuators are typically arranged in antagonistic mode, with both motors of each joint involved in robot motion and stiffness variation. In order to modify the device stiffness, a nonlinear characteristics of the flexible transmissions is needed. This can be realized using either nonlinear springs or linear springs mounted on a nonlinear kinematic transmission. For VSA systems, the paradigm is "design for safety, control for performance" [5]. In particular, the robot can be made more compliant at high speeds and stiffer at low speeds, thus limiting the energy exchange in the first few instants after an unexpected impact. Up to now, the presence of gravity in VSA-based robots has not been treated rigorously, with experimental single-dof devices moving in the horizontal plane or using only a partial gravity compensation - just as in the constant stiffness case. Nonetheless, a large class of VSA-based systems of the antagonistic type has been shown to be feedback equivalent to linear, controllable, and inputoutput decoupled systems [17], [18], with the linearizing outputs being the link position and the device stiffness. Also for this class of flexible devices, such a control property will be useful for removing the dynamic effects of gravity in a complete and efficient way.

In this paper, we present new control results that allow perfect gravity cancellation in dynamic conditions for a variety of robotic systems with flexible transmissions. Based on the general principle of feedback equivalence [19], we design for all cases static state feedback control laws that accurately match the dynamic behavior of the driven links as if they were moving in the absence of gravity. In Sect. II, we consider the case of robots with $n$ elastic joints having constant stiffness and single actuation. In Sect. III, the analysis is extended to transmissions with nonlinear flexibility. The case of antagonistic actuation with variable nonlinear stiffness is handled in Sect. IV. For VSA-based robots, we will be able to impose also a dynamic behavior to the nonlinear stiffness of the device which is identical to that of the no-gravity case. Illustrative simulation results are given in each section.

\section{Robots With Elastic JoINTS}

Consider a robot manipulator having $n$ elastic joints of constant stiffness and $n$ driving motors. Let $\boldsymbol{q}$ and $\boldsymbol{\theta}$ be the $n$-dimensional vectors of link and motor variables. Under the simplifying modeling assumption of Spong [11], and including also viscous effects at the motor and link side, the dynamic model takes the form

$$
\begin{aligned}
\boldsymbol{M}(\boldsymbol{q}) \ddot{\boldsymbol{q}}+\boldsymbol{c}(\boldsymbol{q}, \dot{\boldsymbol{q}})+\boldsymbol{g}(\boldsymbol{q})+\boldsymbol{D}_{q} \dot{\boldsymbol{q}}+\boldsymbol{K}(\boldsymbol{q}-\boldsymbol{\theta}) & =\mathbf{0} \\
\boldsymbol{B} \ddot{\boldsymbol{\theta}}+\boldsymbol{D}_{\theta} \dot{\boldsymbol{\theta}}+\boldsymbol{K}(\boldsymbol{\theta}-\boldsymbol{q}) & =\boldsymbol{\tau},
\end{aligned}
$$

where $\boldsymbol{M}>0$ is the robot inertia matrix, the constant diagonal matrix $\boldsymbol{B}>0$ contains the motor inertias, $\boldsymbol{c}$ is the vector of centrifugal and Coriolis terms, $\boldsymbol{g}$ is the gravity vector, $\boldsymbol{K}>0$ is the diagonal matrix of joint stiffnesses, and $\boldsymbol{D}_{q}$ and $\boldsymbol{D}_{\theta}$ are positive semi-definite diagonal matrices of viscous friction coefficients. In terms of transmission deformation $\boldsymbol{\phi}=\boldsymbol{q}-\boldsymbol{\theta}$, the elastic potential $U_{e}=\frac{1}{2} \boldsymbol{\phi}^{T} \boldsymbol{K} \boldsymbol{\phi}$ associated to (1-2) leads to the linear elasticity torque vector $\tau_{e}$ and constant device stiffness (diagonal) matrix $\boldsymbol{\sigma}$

$$
\boldsymbol{\tau}_{e}=\left(\frac{\partial U_{e}}{\partial \boldsymbol{q}}\right)^{T}=\boldsymbol{K} \boldsymbol{\phi}, \quad \boldsymbol{\sigma}=\frac{\partial \boldsymbol{\tau}_{e}}{\partial \boldsymbol{q}}=\boldsymbol{K} .
$$

Our control goal is to define a (nonlinear) feedback law $\boldsymbol{\tau}=\boldsymbol{\tau}\left(\boldsymbol{q}, \boldsymbol{\theta}, \dot{\boldsymbol{q}}, \dot{\boldsymbol{\theta}}, \boldsymbol{\tau}_{0}\right)$ in (2) such that the behavior of the compensated system matches in suitable coordinates the one of an equivalent model without gravity, i.e.,

$$
\begin{aligned}
\boldsymbol{M}\left(\boldsymbol{q}_{0}\right) \ddot{\boldsymbol{q}}_{0}+\boldsymbol{c}\left(\boldsymbol{q}_{0}, \dot{\boldsymbol{q}}_{0}\right)+\boldsymbol{D}_{q} \dot{\boldsymbol{q}}_{0}+\boldsymbol{K}\left(\boldsymbol{q}_{0}-\boldsymbol{\theta}_{0}\right) & =\mathbf{0} \\
\boldsymbol{B} \ddot{\boldsymbol{\theta}}_{0}+\boldsymbol{D}_{\theta} \dot{\boldsymbol{\theta}}_{0}+\boldsymbol{K}\left(\boldsymbol{\theta}_{0}-\boldsymbol{q}_{0}\right) & =\boldsymbol{\tau}_{0}
\end{aligned}
$$

where a subscript 0 characterizes the variables of the robot without gravity.

It is well known [11] that system (1-2) is exactly linearizable by means of a static state feedback into decoupled chains of four integrators, with $\boldsymbol{q}$ and its first three time derivatives being the linearizing coordinates. The same holds true also for system (3-4). Therefore, thanks to the feedback equivalence principle, by imposing the equality

$$
\boldsymbol{q}(t) \equiv \boldsymbol{q}_{0}(t), \quad \forall t \geq 0
$$

one should obtain the desired result without resorting to the complexity of a complete feedback linearization process. It can be easily verified that this is achieved by choosing the control law as

$$
\tau=\tau_{g}+\tau_{0}
$$

with

$$
\boldsymbol{\tau}_{g}=\boldsymbol{g}(\boldsymbol{q})+\boldsymbol{D}_{\theta} \boldsymbol{K}^{-1} \dot{\boldsymbol{g}}(\boldsymbol{q})+\boldsymbol{B} \boldsymbol{K}^{-1} \ddot{\boldsymbol{g}}(\boldsymbol{q}),
$$

where

$$
\begin{aligned}
\dot{\boldsymbol{g}}(\boldsymbol{q})= & \frac{\partial \boldsymbol{g}(\boldsymbol{q})}{\partial \boldsymbol{q}} \dot{\boldsymbol{q}} \\
\ddot{\boldsymbol{g}}(\boldsymbol{q})= & \frac{\partial \boldsymbol{g}(\boldsymbol{q})}{\partial \boldsymbol{q}} \boldsymbol{M}^{-1}(\boldsymbol{q})\left(\boldsymbol{K}(\boldsymbol{\theta}-\boldsymbol{q})-\boldsymbol{c}(\boldsymbol{q}, \dot{\boldsymbol{q}})-\boldsymbol{g}(\boldsymbol{q})-\boldsymbol{D}_{q} \dot{\boldsymbol{q}}\right) \\
& +\sum_{i=1}^{n} \frac{\partial^{2} \boldsymbol{g}(\boldsymbol{q})}{\partial \boldsymbol{q} \partial q_{i}} \dot{\boldsymbol{q}}_{i},
\end{aligned}
$$

provided that matched initial conditions hold at time $t=0$ :

$$
\begin{aligned}
\boldsymbol{q}(0) & =\boldsymbol{q}_{0}(0) & \ddot{\boldsymbol{q}}(0) & =\ddot{\boldsymbol{q}}_{0}(0) \\
\dot{\boldsymbol{q}}(0) & =\dot{\boldsymbol{q}}_{0}(0) & \boldsymbol{q}^{[3]}(0) & =\boldsymbol{q}_{0}^{[3]}(0),
\end{aligned}
$$

with the notation $\boldsymbol{q}^{[i]}=d^{i} \boldsymbol{q} / d t^{i}$. 
The expression of the control law (5-6) is obtained by imposing $\boldsymbol{q}^{[4]}(0)=\boldsymbol{q}_{0}^{[4]}(0)$, taking into account that the identities (7) are automatically enforced for all $t \geq 0$. A notable feature is that this control law can be computed in closed form. Moreover, in any static condition, i.e., with $\dot{\boldsymbol{q}}=\ddot{\boldsymbol{q}}=\mathbf{0}$, the gravity cancellation torque becomes $\boldsymbol{\tau}_{g}=\boldsymbol{g}(\boldsymbol{q})$, as expected. Instead, in dynamic conditions $\boldsymbol{\tau}_{g}$ includes terms that are proportional to the inverse of the joint stiffness $\boldsymbol{K}$. Thus, the more rigid are the transmissions the less extra dynamic torque is needed for gravity cancellation. We remark that, despite of the need of inverting the robot inertia matrix $\boldsymbol{M}(\boldsymbol{q})$, the gravity cancellation torque (6) is much simpler than the expression of a feedback linearization control law, which would involve also the time derivatives of the model terms $\boldsymbol{M}(\boldsymbol{q})$ and $\boldsymbol{c}(\boldsymbol{q}, \dot{\boldsymbol{q}})$ up to the second order.

There are indeed differences in the state behavior between the gravity-free system (3-4) and system (1-2) under the gravity cancellation control law (5-6). While the two systems will evolve in an identical way when looking at the linearizing coordinates $\boldsymbol{q}(t) \equiv \boldsymbol{q}_{0}(t)$, the inverse mappings of this evolution in terms of the respective motor variables will still be different. In fact, since the initial matching conditions (7) will be satisfied also for all $t \geq 0$, it is

$$
\begin{aligned}
& \boldsymbol{\theta}(t)=\boldsymbol{\theta}_{0}(t)+\boldsymbol{K}^{-1} \boldsymbol{g}(\boldsymbol{q}(t)) \neq \boldsymbol{\theta}_{0}(t) \\
& \dot{\boldsymbol{\theta}}(t)=\dot{\boldsymbol{\theta}}_{0}(t)+\boldsymbol{K}^{-1} \frac{\partial \boldsymbol{g}(\boldsymbol{q}(t))}{\partial \boldsymbol{q}} \dot{\boldsymbol{q}}(t) \neq \dot{\boldsymbol{\theta}}_{0}(t) .
\end{aligned}
$$

This should not be surprising from a physical point of view: the gravity-loaded robot needs the presence of a deformation $\boldsymbol{q}-\boldsymbol{\theta} \neq \boldsymbol{q}-\boldsymbol{\theta}_{0}$ that dynamically balances the gravity on the link side. The control law (5-6) will only cancel the effects on the link (output) motion, which is what we actually need during robot interaction with the environment/human.

The torque input $\tau_{0}$ in (5) can be chosen according to the task, e.g., for a torque-based robot reaction to detected collisions as in [2], or for a regulation task to a constant link position $\boldsymbol{q}_{d}$.

\section{A. Simulation results}

To illustrate the performance of the control law (5-6), it is sufficient to compare the behavior of a single link with an elastic joint in the absence of gravity and that under gravity but with dynamic gravity cancellation. In this case, the link inertia is a constant scalar $M$ and the gravity term is given by $g(q)=m d g_{0} \sin q$, where $m$ is the mass of the link, $d$ is the distance of its center of mass from the joint, and $g_{0}$ is the gravity acceleration. The dynamic gravity cancellation term $\tau_{g}$ in (6) is

$$
\begin{aligned}
\tau_{g}=m d g_{0}\{ & \left(1-\frac{B}{K} \dot{q}^{2}\right) \sin q-\frac{B}{M} \frac{m d g_{0}}{K} \sin q \cos q \\
& \left.+\frac{M D_{\theta}-B D_{q}}{K M} \dot{q} \cos q+\frac{B}{M}(\theta-q) \cos q\right\} .
\end{aligned}
$$

Using $M=8.333, B=50\left[\mathrm{~N} \cdot \mathrm{mm} \cdot \mathrm{s}^{2} / \mathrm{rad}\right], m=0.1[\mathrm{~kg}]$, $d=250[\mathrm{~mm}], D_{q}=0.1, D_{\theta}=1[\mathrm{~N} \cdot \mathrm{mm} \cdot \mathrm{s} / \mathrm{rad}]$, and $K=100[\mathrm{~N} \cdot \mathrm{mm} / \mathrm{rad}]$ as data, we simulated the two systems starting at rest from the downward equilibrium, and applying

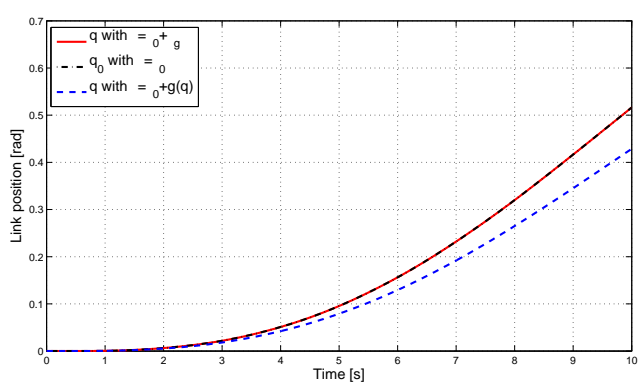

(a)

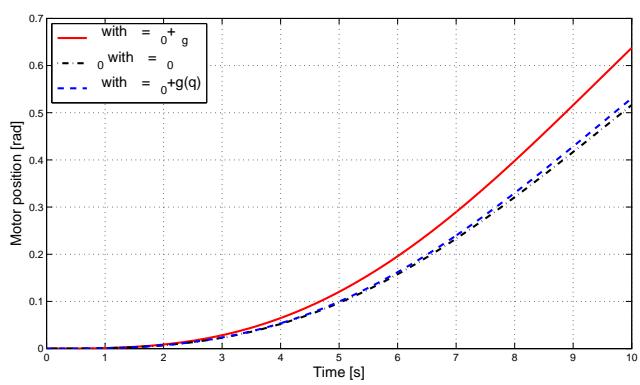

(b)

Fig. 1. Comparison of link (a) and motor (b) position for a single elastic joint without gravity under $\tau_{0}$ [dot-dashed, black], and with gravity under $\tau_{0}$ and a link-based compensation $g(q)$ [dashed, blue] or under $\tau_{0}$ and the dynamic cancellation law $\tau_{g}$ in (8) [continuous, red]

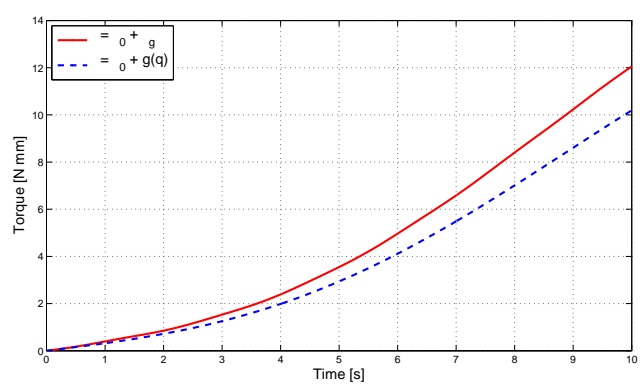

Fig. 2. Total applied torques with $g(q)$ only [dashed, blue] and with $\tau_{g}$ in (8) [continuous, red] for the motion of Fig. 1

the open-loop torque $\tau_{0}=\sin 0.1 \pi t$ for $T=10 \mathrm{~s}$. Figure 1 shows the obtained evolution of the link (a) and motor (b) angles in the absence or presence of gravity. For the latter case, we compare also the use of a simpler link-based compensation $g(q)$ in place of the dynamic cancellation $\tau_{g}$ given by (8). From Fig. 1(a), it can be seen that $q(t)=q_{0}(t)$ exactly in the case of dynamic cancellation, while an error is present when using $g(q)$. On the other hand, $\theta(t) \neq \theta_{0}(t)$ (both with dynamic cancellation and link-based compensation) despite the initial states of the systems with and without gravity were fully matched at $t=0$, with no initial joint deformation (see Fig. 1(b)). The total torques (i.e., including $\tau_{0}$ ) for the link-based gravity compensation and for its perfect cancellation are reported in Fig. 2, showing that the dynamic torque contribution is indeed non-negligible. 


\section{JoINTS WITH NONLINEAR FLEXIBILITY}

In this section, we extend the previous analysis to the case of transmissions with nonlinear flexibility [20]. For the sake of simplicity, only a single dof will be considered, but the generalization to multi-dof systems is straightforward. Using the same notation of Sec. II, we assume that a potential energy $U_{e}(\phi) \geq 0$ is associated to the deformation $\phi=q-\theta$, so that the flexibility torque $\tau_{e}=\partial U_{e} / \partial q=\tau_{e}(\phi)$ is a nonlinear function of $\phi$ and the stiffness $\sigma=\partial \tau_{e} / \partial q=\sigma(\phi)$ will be non-constant.

The dynamic model of a single link moving under gravity and driven through such a flexible transmission is then

$$
\begin{aligned}
M \ddot{q}+D_{q} \dot{q}+g(q)+\tau_{e}(\phi) & =0 \\
B \ddot{\theta}+D_{\theta} \dot{\theta}-\tau_{e}(\phi) & =\tau .
\end{aligned}
$$

We wish to define a feedback law $\tau=\tau\left(q, \theta, \dot{q}, \dot{\theta}, \tau_{0}\right)$ in (10) so as to match the behavior of some variable of the model without gravity

$$
\begin{aligned}
M \ddot{q}_{0}+D_{q} \dot{q}_{0}+\tau_{e}\left(\phi_{0}\right) & =0 \\
B \ddot{\theta}_{0}+D_{\theta} \dot{\theta}_{0}-\tau_{e}\left(\phi_{0}\right) & =\tau_{0} .
\end{aligned}
$$

It is easy to verify that the nonlinear systems (9-10) and (11-12) are exactly linearizable by means of a static state feedback into a chain of four integrators, with $q$ and its first three time derivatives as linearizing coordinates. Therefore, the two systems are feedback equivalent, and the solution to our problem is obtained by imposing $q(t)=q_{0}(t)$ for all $t \geq 0$. In particular, from $q^{[4]}=q_{0}^{[4]}$ we get

$$
\begin{aligned}
\tau= & g(q)+\frac{D_{\theta}}{\sigma(\phi)} \dot{g}(q)+\frac{B}{\sigma(\phi)} \ddot{g}(q) \\
& +\frac{\sigma(\phi)-\sigma\left(\phi_{0}\right)}{\sigma(\phi)}\left((B+M) \ddot{q}+\left(D_{q}+D_{\theta}\right) \dot{q}\right) \\
& +\frac{B}{\sigma(\phi)}\left(\frac{\partial \sigma(\phi)}{\partial \phi} \dot{\phi}^{2}-\frac{\partial \sigma\left(\phi_{0}\right)}{\partial \phi_{0}} \dot{\phi}_{0}^{2}\right)+\frac{\sigma\left(\phi_{0}\right)}{\sigma(\phi)} \tau_{0} \\
= & \tau_{g}+\alpha_{g} \tau_{0},
\end{aligned}
$$

where $\ddot{q}$ (to be used also in $\ddot{g}(q)$ ) is computed from (9) as

$$
\ddot{q}=-\frac{1}{M}\left(D_{q} \dot{q}+g(q)+\tau_{e}(\phi)\right) .
$$

In addition, the initial matching requires

$$
\begin{aligned}
q(0) & =q_{0}(0) & \ddot{q}(0) & =\ddot{q}_{0}(0) \\
\dot{q}(0) & =\dot{q}_{0}(0) & q^{[3]}(0) & =q_{0}^{[3]}(0) .
\end{aligned}
$$

The expression (13) collapses into (5-6) for a transmission with constant stiffness $\sigma=K$. However, differently from the case of linear elasticity, the control law (13) contains terms that require the knowledge of the deformation $\phi_{0}=q-\theta_{0}$, and of its rate $\dot{\phi}_{0}$, pertaining to the model without gravity. Also, the torque $\tau_{0}$ applied in the gravity-free case needs now to be scaled by the factor $\alpha_{g}=\sigma\left(\phi_{0}\right) / \sigma(\phi)$.

The value $\phi_{0}$ is computed by solving the nonlinear equation $\tau_{e}\left(\phi_{0}\right)=-M \ddot{q}-D_{q} \dot{q}$, which is obtained from (11) by taking into account the first three identities in (14). Using (9), the right-hand side can be written as a function of the state (actually, of the configuration variables only) of the gravityloaded system as

$$
\tau_{e}\left(\phi_{0}\right)=g(q)+\tau_{e}(\phi)=a(q, \theta) .
$$

Equation (15) needs to be solved at each time $t \geq 0$, as a function of the current system state. As a representative example, consider a flexible joint transmission with associated potential given by $U_{e}=\frac{1}{2} K \phi^{2}+\frac{1}{4} K_{c} \phi^{4}$, with $K>0$ and $K_{c}>0$ [21]. The flexibility torque is a cubic function of $\phi$ and the stiffness has a quadratic dependence:

$$
\tau_{e}(\phi)=K \phi+K_{c} \phi^{3}, \quad \sigma(\phi)=K+3 K_{c} \phi^{2} .
$$

At a given $(q, \theta)$, equation (15) results in the cubic equation $K_{c} \phi_{0}^{3}+K \phi_{0}-a(q, \theta)=0$, which has always two complex roots and one real (positive or negative) root, thanks to the positivity of $K$ and $K_{c}$. The real root is given by

$$
\phi_{0}=\sqrt[3]{\frac{1}{2} \frac{a(q, \theta)}{K_{c}}+b(q, \theta)}+\sqrt[3]{\frac{1}{2} \frac{a(q, \theta)}{K_{c}}-b(q, \theta)},
$$

where $b(q, \theta)=\sqrt{\frac{1}{27}\left(\frac{K}{K_{c}}\right)^{3}+\frac{1}{4}\left(\frac{a(q, \theta)}{K_{c}}\right)^{2}}>0$. For more general stiffness profiles, a solution to (15) should be searched numerically.

Once $\phi_{0}$ has been found, the value of $\dot{\phi}_{0}$ that appears in the control law (13) is obtained by time differentiation of (15) (or, equivalently, from the fourth identity in (14)) as

$$
\dot{\phi}_{0}=\frac{1}{\sigma\left(\phi_{0}\right)}\left(\sigma(\phi) \dot{\phi}+\frac{\partial g(q)}{\partial q} \dot{q}\right) \text {. }
$$

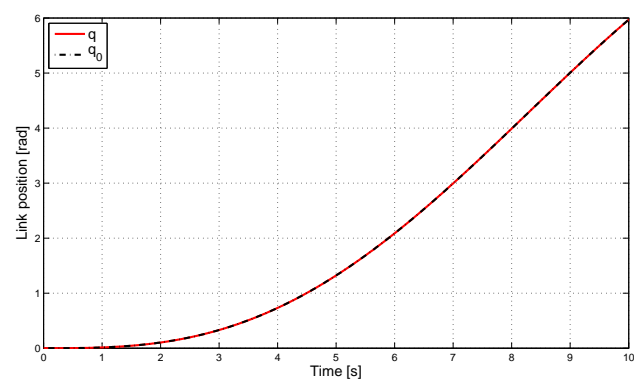

(a)

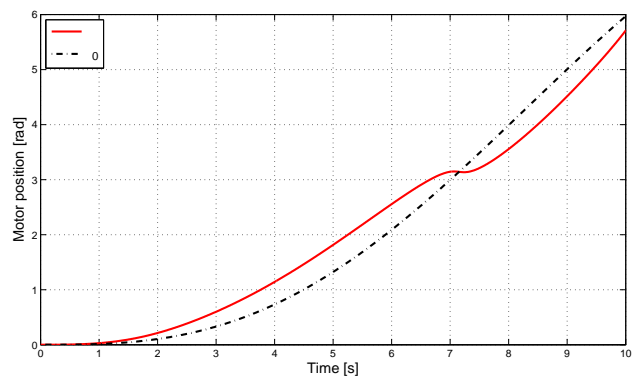

(b)

Fig. 3. Comparison of link (a) and motor (b) position for a single nonlinear flexible joint without gravity under $\tau_{0}$ [dot-dashed, black], and with gravity under the dynamic cancellation law (13) [continuous, red] 
As a result, the gravity cancellation control law (13) can be computed in closed form from full state measurements in the case of cubic stiffness (and for other simple nonlinear dependences). Note that for multi-dof robots with nonlinear flexible joints one needs to solve $n$ similar equations of the form (15), whereas (17) is replicated component-wise.

\section{A. Simulation results}

We simulated a joint with cubic flexibility torque $\tau_{e}(\phi)$ having $K=100[\mathrm{~N} \cdot \mathrm{mm} / \mathrm{rad}]$ and $K_{c}=500[\mathrm{~N} \cdot \mathrm{mm} / \mathrm{rad}]$. With these data, the joint stiffness $\sigma(\phi)$ increases by about $45 \%$ w.r.t. its value at $\phi=0$ when the joint deformation is $|\phi|=0.18$ [rad]. All other model parameters, the initial conditions, and the open-loop input torque are the same as in Sect. II-A. Figure 3 shows the evolution of the link (a) and motor (b) angles obtained in the absence or in the presence of gravity under the dynamic gravity cancellation law (13).

\section{VARiable Stiffness Joints with Antagonistic ACTUATION}

Progressing in the generalization of the dynamic gravity cancellation approach, we consider in this section the case of joints with (actuated) variable stiffness. Use of variable nonlinear stiffness actuation is highly recommended for safe pHRI, with the antagonistic arrangement of two motors for each joint as the most common realization [13], [15], [16]. For the sake of notational simplicity, consider a single link under gravity driven by a VSA system. The dynamic model is expressed in terms of three generalized coordinates, $q$ for the link position, and $\theta_{1}$ and $\theta_{2}$ for the position of the two motors. Let $\phi_{i}=q-\theta_{i}, i=1,2$, be the deformations of the transmissions at the two sides of the joint. We have

$$
\begin{aligned}
M \ddot{q}+D_{q} \dot{q}+g(q)+\tau_{e}\left(\phi_{1}\right)+\tau_{e}\left(\phi_{2}\right) & =0 \\
B \ddot{\theta}_{1}+D_{\theta} \dot{\theta}_{1}-\tau_{e}\left(\phi_{1}\right) & =\tau_{1} \\
B \ddot{\theta}_{2}+D_{\theta} \dot{\theta}_{2}-\tau_{e}\left(\phi_{2}\right) & =\tau_{2},
\end{aligned}
$$

where $\tau_{1}$ and $\tau_{2}$ are the torques supplied by the two motors. Without loss of generality, we have assumed in (18-20) a full symmetry for the two actuation/transmission systems. Accordingly, the total stiffness $\sigma_{t}$ of the device is given by the separable function

$$
\sigma_{t}\left(\phi_{1}, \phi_{2}\right)=\frac{\partial\left(\tau_{e}\left(\phi_{1}\right)+\tau_{e}\left(\phi_{2}\right)\right)}{\partial q}=\sigma\left(\phi_{1}\right)+\sigma\left(\phi_{2}\right) .
$$

As before, the target behavior is specified by a dynamic system of the same form of (18-20), but with $g(q) \equiv 0$ and all its variables labeled by a 0 subscript.

Since the system has two inputs, according to the feedback equivalence principle, we should determine two independent system output functions that play the role of linearizing coordinates in a feedback linearization scheme. Based on the results in [18], these two outputs are the link position $q$ and the total stiffness $\sigma_{t}$. In fact, differentiating (18) w.r.t. time gives

$$
M q^{[3]}+D_{q} \ddot{q}+\dot{g}(q)+\sigma\left(\phi_{1}\right) \dot{\phi}_{1}+\sigma\left(\phi_{2}\right) \dot{\phi}_{2}=0 .
$$

Differentiating once more, using (19-20), and rearraging terms, we obtain

$$
\begin{aligned}
& M q^{[4]}+D_{q} q^{[3]}+\ddot{g}(q)+\frac{\partial \sigma\left(\phi_{1}\right)}{\partial \phi_{1}} \dot{\phi}_{1}^{2}+\frac{\partial \sigma\left(\phi_{2}\right)}{\partial \phi_{2}} \dot{\phi}_{2}^{2}+\sigma_{t} \ddot{q} \\
& =\sigma\left(\phi_{1}\right) \ddot{\theta}_{1}+\sigma\left(\phi_{2}\right) \ddot{\theta}_{2} \\
& =\frac{1}{B}\left(\begin{array}{ll}
\sigma\left(\phi_{1}\right) & \sigma\left(\phi_{2}\right)
\end{array}\right)\left(\begin{array}{c}
\tau_{1}+\tau_{e}\left(\phi_{1}\right)-D_{\theta} \dot{\theta}_{1} \\
\tau_{2}+\tau_{e}\left(\phi_{2}\right)-D_{\theta} \dot{\theta}_{2}
\end{array}\right) .
\end{aligned}
$$

Similarly, by differentiating (21) w.r.t. time, we have

$$
\dot{\sigma}_{t}=\frac{\partial \sigma\left(\phi_{1}\right)}{\partial \phi_{1}} \dot{\phi}_{1}+\frac{\partial \sigma\left(\phi_{2}\right)}{\partial \phi_{2}} \dot{\phi}_{2}
$$

and, by rearraging terms and using again (19-20),

$$
\begin{aligned}
& \ddot{\sigma}_{t}=\frac{\partial^{2} \sigma\left(\phi_{1}\right)}{\partial \phi_{1}^{2}} \dot{\phi}_{1}^{2}+\frac{\partial^{2} \sigma\left(\phi_{2}\right)}{\partial \phi_{2}^{2}} \dot{\phi}_{2}^{2}+\left(\frac{\partial \sigma\left(\phi_{1}\right)}{\partial \phi_{1}}+\frac{\partial \sigma\left(\phi_{2}\right)}{\partial \phi_{2}}\right) \ddot{q} \\
& =\frac{\partial \sigma\left(\phi_{1}\right)}{\partial \phi_{1}} \ddot{\theta}_{1}+\frac{\partial \sigma\left(\phi_{2}\right)}{\partial \phi_{2}} \ddot{\theta}_{2} \\
& =\frac{1}{B}\left(\begin{array}{ll}
\frac{\partial \sigma\left(\phi_{1}\right)}{\partial \phi_{1}} & \frac{\partial \sigma\left(\phi_{2}\right)}{\partial \phi_{2}}
\end{array}\right)\left(\begin{array}{l}
\tau_{1}+\tau_{e}\left(\phi_{1}\right)-D_{\theta} \dot{\theta}_{1} \\
\tau_{2}+\tau_{e}\left(\phi_{2}\right)-D_{\theta} \dot{\theta}_{2}
\end{array}\right) .
\end{aligned}
$$

It can be shown that the decoupling matrix associated to the output vector $\left(q, \sigma_{t}\right)$ is proportional to the matrix

$$
\mathcal{A}\left(\phi_{1}, \phi_{2}\right)=\left(\begin{array}{cc}
\sigma\left(\phi_{1}\right) & \sigma\left(\phi_{2}\right) \\
\frac{\partial \sigma\left(\phi_{1}\right)}{\partial \phi_{1}} & \frac{\partial \sigma\left(\phi_{2}\right)}{\partial \phi_{2}}
\end{array}\right),
$$

which is generically nonsingular, except when $\theta_{1}=\theta_{2}$ (a condition that can be always avoided by suitably precharging the actuation system). Therefore, the outputs $q$, together with its first three derivatives, and $\sigma_{t}$, with its first derivative, are linearizing coordinates for system (18-20).

Comparing the expressions (23) and (25) with those of the gravity-free case (with a 0 subscript), the solution to the problem of dynamic gravity cancellation is given by the control torques $\tau_{1}$ and $\tau_{2}$

$$
\begin{gathered}
\left(\begin{array}{c}
\tau_{1} \\
\tau_{2}
\end{array}\right)=\left(\begin{array}{c}
D_{\theta} \dot{\theta}_{1}-\tau_{e}\left(\phi_{1}\right) \\
D_{\theta} \dot{\theta}_{2}-\tau_{e}\left(\phi_{2}\right)
\end{array}\right)+\mathcal{A}^{-1}\left(\phi_{1}, \phi_{2}\right) \cdot \\
\left\{\mathcal{A}\left(\phi_{10}, \phi_{20}\right)\left(\left(\begin{array}{c}
\tau_{10} \\
\tau_{20}
\end{array}\right)+\left(\begin{array}{c}
\tau_{e}\left(\phi_{10}\right)-D_{\theta} \dot{\theta}_{10} \\
\tau_{e}\left(\phi_{20}\right)-D_{\theta} \dot{\theta}_{20}
\end{array}\right)\right)\right. \\
\left.+B\left(\begin{array}{c}
\ddot{g}(q)+\sum_{i=1}^{2}\left(\frac{\partial \sigma\left(\phi_{i}\right)}{\partial \phi_{i}} \dot{\phi}_{i}^{2}-\frac{\partial \sigma\left(\phi_{i 0}\right)}{\partial \phi_{i 0}} \dot{\phi}_{i 0}^{2}\right) \\
\sum_{i=1}^{2}\left(\frac{\partial \sigma\left(\phi_{i}\right)}{\partial \phi_{i}}-\frac{\partial \sigma\left(\phi_{i 0}\right)}{\partial \phi_{i 0}}\right) \ddot{q} \\
+\sum_{i=1}^{2}\left(\frac{\partial^{2} \sigma\left(\phi_{i}\right)}{\partial \phi_{i}^{2}} \dot{\phi}_{i}^{2}-\frac{\partial^{2} \sigma\left(\phi_{i 0}\right)}{\partial \phi_{i 0}^{2}} \dot{\phi}_{i 0}^{2}\right)
\end{array}\right)\right\}
\end{gathered}
$$

where the link acceleration $\ddot{q}$ (to be used also in $\ddot{g}(q)$ ) is computed from (18) as

$$
\ddot{q}=-\frac{1}{M}\left(D_{q} \dot{q}+g(q)+\tau_{e}\left(\phi_{1}\right)+\tau_{e}\left(\phi_{2}\right)\right) .
$$

In addition, an initial state matching given by

$q(0)=q_{0}(0) \quad \dot{q}(0)=\dot{q}_{0}(0) \quad \ddot{q}(0)=\ddot{q}_{0}(0) \quad q^{[3]}(0)=q_{0}^{[3]}(0)$ 
and

$$
\begin{aligned}
\sigma_{t}(0)=\sigma_{t}\left(\phi_{1}(0), \phi_{2}(0)\right) & =\sigma_{t}\left(\phi_{10}(0), \phi_{20}(0)\right)=\sigma_{t 0}(0) \\
\dot{\sigma}_{t}(0) & =\dot{\sigma}_{t 0}(0)
\end{aligned}
$$

should hold between the gravity-loaded and the gravity-free system. Note that the above identities will hold for all $t \geq 0$ thanks to the chosen control law.

The control law (26) physically replaces all terms that are affected by gravity (motor variables, flexible deformation torques, partial derivatives of the stiffness functions) with those of the gravity-free target system. For the considered single-dof VSA-based joint, the dynamic gravity cancellation law is very similar to a feedback linearization law from the point of view of complexity. However, these two controllers will differ consistently when considering multi-dof VSA robotic systems -in much the same way as for the case of single actuation of linear or nonlinear flexible joints.

Beside measurements of the state of the gravity-loaded system, in order to evaluate (26) we need also knowledge of the deformations $\phi_{i 0}, i=1,2$, and of their rates $\dot{\phi}_{i 0}$, $i=1,2$, pertaining to the target system without gravity. Note that from $\phi_{i 0}$, we directly obtain also $\theta_{i 0}=q-\phi_{i 0}$. Similarly to Sect. III, the deformations $\phi_{10}$ and $\phi_{20}$ are determined by solving the coupled system of two nonlinear equations

$$
\begin{aligned}
\tau_{e}\left(\phi_{10}\right)+\tau_{e}\left(\phi_{20}\right) & =-M \ddot{q}-D_{q} \dot{q}=a_{1}\left(q, \theta_{1}, \theta_{2}\right) \\
\sigma\left(\phi_{10}\right)+\sigma\left(\phi_{20}\right) & =\sigma_{t}\left(q, \theta_{1}, \theta_{2}\right),
\end{aligned}
$$

where the right-hand sides of (27) are expressed in terms of current state measurements using (18) and (21). Due to the symmetry, if $\left(\phi_{a}, \phi_{b}\right)$ is a solution of (27) then $\left(\phi_{b}, \phi_{a}\right)$ is a solution as well.

In general, system (27) needs to be solved numerically. Some additional insight is provided in the case of cubic flexibility torques, see (16). We have then

$$
\begin{aligned}
K\left(\phi_{10}+\phi_{20}\right)+K_{c}\left(\phi_{10}^{3}+\phi_{20}^{3}\right) & =a_{1}\left(q, \theta_{1}, \theta_{2}\right) \\
2 K+3 K_{c}\left(\phi_{10}^{2}+\phi_{20}^{2}\right) & =\sigma_{t}\left(q, \theta_{1}, \theta_{2}\right) .
\end{aligned}
$$

Since by definition

$$
\frac{\sigma_{t}-2 K}{3 K_{c}}:=R^{2} \geq 0,
$$

the solutions to equation (29) can be parametrized by a scalar $\alpha \in[0,2 \pi)$ as $\phi_{10}=R \cos \alpha$ and $\phi_{20}=R \sin \alpha$. Replacing these in (28) yields the single trigonometric equation in $\alpha$

$$
(\cos \alpha+\sin \alpha)+\frac{\sigma_{t}-2 K}{3 K}\left(\cos ^{3} \alpha+\sin ^{3} \alpha\right)=\frac{a_{1}}{K R} .
$$

Figure 4 shows a plot of one of the two branches of the expression on the left-hand side of (30), obtained for $K=100$, $K_{c}=500$, and $\sigma_{t}=220$. The horizontal line corresponds to the case $a_{1}=10$, and the associated root $\alpha$ provides the solution $\phi_{10}=0.1136$ and $\phi_{20}=-0.0209$ [rad]. It can be seen that equation (30) is sufficiently smooth, and thus easily solvable by a numerical root finder (e.g., the fzero routine of Matlab). Assume now that the device stiffness $\sigma_{t}$ can be changed within the interval $(2 K, 4 K)$, i.e., from its minimum physical value to a $100 \%$ increase. It can be shown that a

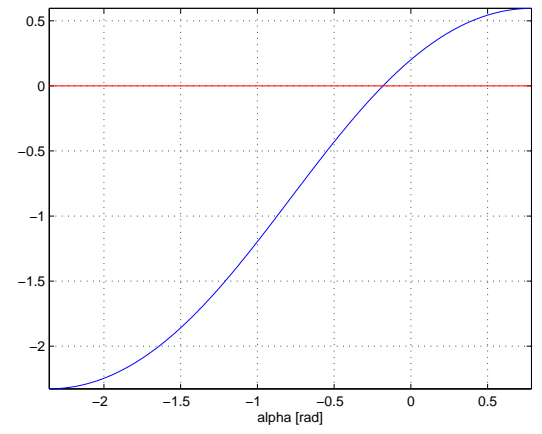

Fig. 4. A typical functional form of eq. (30) and a possible solution

pair of solutions $\alpha$ to (30) always exist in this interval for $\sigma_{t}$, provided that $\left|a_{1}\right|<\sqrt{2} K R\left[1+0.5\left(\sigma_{t}-2 K\right) /(3 K)\right]$. It should be stressed that the existence of pairs of solutions is not a source of problems. In fact, system (27) will be solved at every (discretized) instant $t \geq 0$. Once a specific solution has been chosen at $t=0$, the process is repeated on line and a local numerical search around the previous solution generates a single update.

Finally, having determined $\phi_{10}$ and $\phi_{20}$, their rates are obtained by time differentiation of (27) as

$$
\begin{aligned}
\left(\begin{array}{c}
\dot{\phi}_{10} \\
\dot{\phi}_{20}
\end{array}\right) & =\mathcal{A}^{-1}\left(\phi_{10}, \phi_{20}\right)\left(\begin{array}{c}
\left.-M q^{[3]}-D_{q} \ddot{q}\right) \\
\dot{\sigma}_{t}
\end{array}\right) \\
& =\mathcal{A}^{-1}\left(\phi_{10}, \phi_{20}\right)\left(\begin{array}{c}
\sigma\left(\phi_{1}\right) \dot{\phi}_{1}+\sigma\left(\phi_{2}\right) \dot{\phi}_{2}+\frac{\partial g(q)}{\partial q} \dot{q} \\
\frac{\partial \sigma\left(\phi_{1}\right)}{\partial \phi_{1}} \dot{\phi}_{1}+\frac{\partial \sigma\left(\phi_{2}\right)}{\partial \phi_{2}} \dot{\phi}_{2}
\end{array}\right),
\end{aligned}
$$

where (22) and (24) have been used to express all quantities in terms of the original VSA system state only.

\section{A. Simulation results}

We have simulated the dynamic gravity cancellation law (26) for a symmetric antagonistic joint with cubic flexibility torques, using the numerical data of Sec. III-A duplicated as needed. In the present case, the input torques $\tau_{10}$ and $\tau_{20}$ have been chosen of the bang-bang type as in Fig. 5(c). Figure 5 shows the validity of the proposed scheme: both the link position (a) and the device stiffness (b) have identical evolutions in the absence of gravity and when gravity is present but dynamically canceled. Note that the stiffness variation during motion is as large as $2.5[\mathrm{Nm} / \mathrm{rad}]$. The total applied torques are shown in Fig. 6.

\section{B. The VSA-II Variable Stiffness Joint}

We report also a numerical result on gravity cancellation for the VSA-II experimental device developed at the University of Pisa [16] and sketched in Fig. 7. The VSA-II is based on a bi-directional antagonistic arrangement of two motors driving a single joint through a nonlinear flexible transmission system that uses pairs of 4-bar mechanisms (the so-called Grashof neutral linkage) with linear springs.

The VSA-II dynamic model takes the form (18-20), with

$$
\tau_{e}\left(\phi_{i}\right)=2 K \beta\left(\phi_{i}\right) \frac{\partial \beta\left(\phi_{i}\right)}{\partial \phi_{i}}, \quad i=1,2,
$$




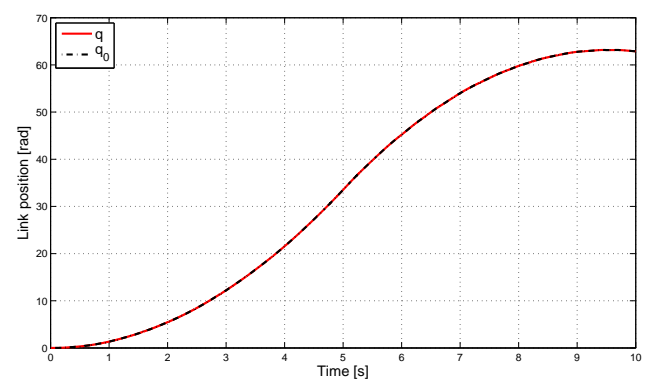

(a)

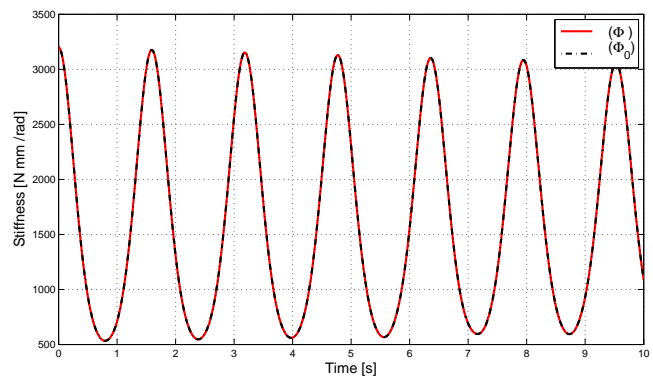

(b)

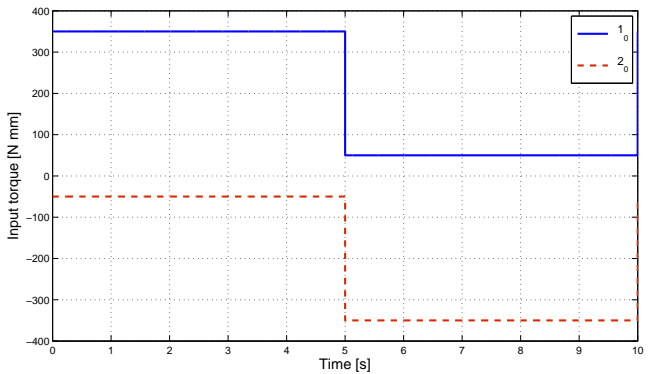

(c)

Fig. 5. Comparison of link position (a) and device stiffness (b) for a variable stiffness antagonistic joint with cubic flexibility torques without gravity [dot-dashed, black], and with gravity under the dynamic cancellation law (26) [continuous, red] when the bang-bang torque inputs $\tau_{10}$ and $\tau_{10}$ (c) are applied

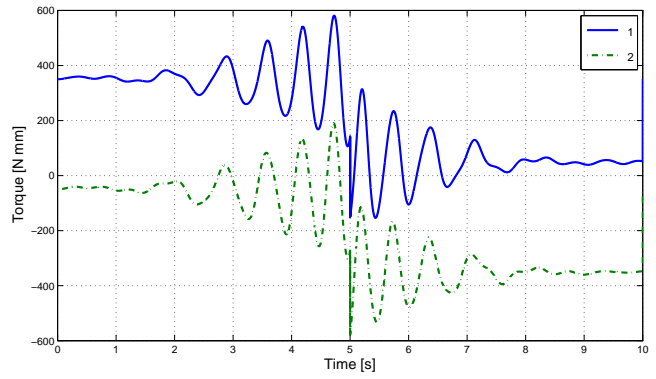

Fig. 6. Total applied torques (26) for the link motion and device stiffness evolution of Fig. 5

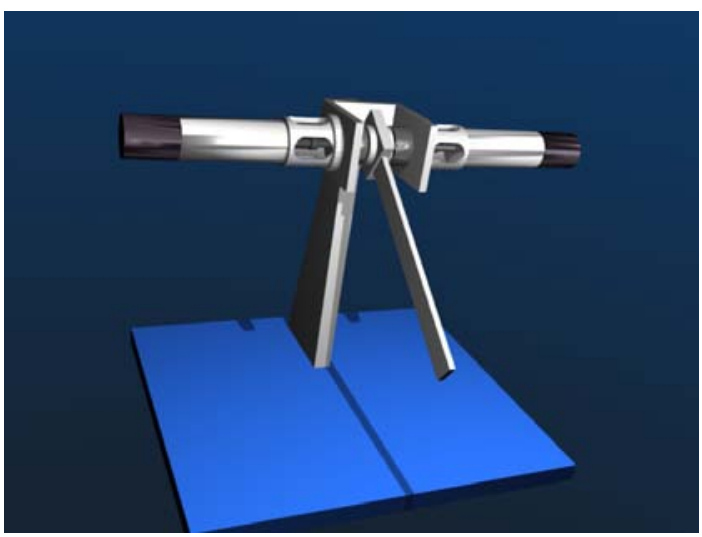

Fig. 7. The VSA-II variable stiffness actuator moving a link under gravity

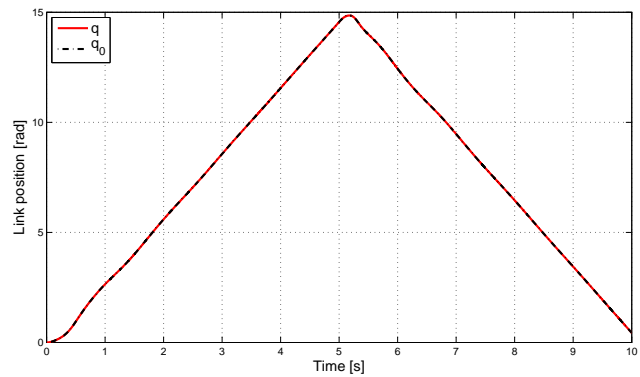

(a)

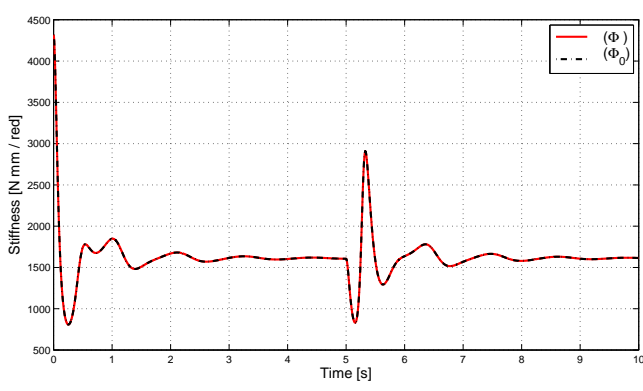

(b)

Fig. 8. Comparison of link position (a) and device stiffness (b) for the VSAII without gravity [dot-dashed, black], and with gravity under the dynamic cancellation law [continuous, red] when the bang-bang torque inputs $\tau_{10}$ and $\tau_{10}$ of Fig. 5(c) are applied

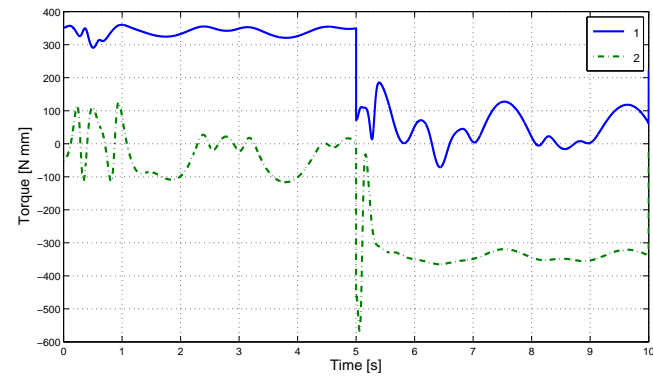

Fig. 9. Total applied torques for the link motion and device stiffness evolution of Fig. 8 
where $K$ is the constant stiffness of each of the two torsional elastic springs and

$$
\beta\left(\phi_{i}\right)=\arcsin \left(C \sin \left(\frac{\phi_{i}}{2}\right)\right)-\frac{\phi_{i}}{2}, \quad i=1,2,
$$

being $C>1$ a geometric parameter of the 4-bar mechanisms. Due to this arrangement, the dynamic gravity cancellation law for the VSA-II is a particular instance of eq. (26).

Figure 8 shows the obtained evolution when using the same open-loop torque input of Fig. 5(c) and the numerical data from [16], [18]. The total applied torques are reported in Fig. 9.

\section{CONCLUSIONS}

We have considered the problem of perfect dynamic cancellation of gravity effects acting on the link motion of robot manipulators having flexible transmissions. The cases of flexible transmissions with constant, nonlinear, and variable nonlinear stiffness with antagonistic actuation have been analyzed. Based on the feedback equivalence principle, state feedback control laws have been designed that let the system outputs behave as those of a reference model where gravity is absent. In the case of VSA-based manipulators, this includes also intervention on the device stiffness. While dynamic gravity cancellation involves in general the on-line computation of inertial terms, the presented control laws are much simpler than those needed for feedback linearization. Control solutions are obtained either in closed algebraic form or by using simple numerical techniques. In particular, the parallel simulation of the gravity-free system to be matched is never required.

The presented results can be used for different control purposes. For set-point regulation tasks of robots with elastic joints, a PD-type control law on the motor position error can be designed on top of the gravity cancellation law, and its global asymptotic stability can be shown without the need of a strictly positive lower bound neither on the proportional gain nor on the joint stiffness [22]. Regulation controllers should be more easily obtained in this way also for VSA-based manipulators, where the link position as well as the device stiffness need to be asymptotically stabilized to a desired constant value. Moreover, the proposed dynamic gravity cancellation is useful in safe physical human-robot interaction. In general, unexpected collisions may occur at any time during motion and the compliant robot should react as soon as the impact is detected (e.g., with a sensorless residual-based method as in [2]). Through the permanent cancellation of the gravitational loads on the robot links, a physical torque-based reaction strategy can be designed so that the controlled robot rapidly flees away from the danger area in a gravity-unbiased dynamic fashion. This subject is currently under investigation.

\section{ACKNOWLEDGMENTS}

This work has been funded by the MIUR project PRIN 2007 SICURA.

\section{REFERENCES}

[1] J. Heinzmann and A. Zelinsky, "Quantitative safety guarantees for physical human-robot interaction," Int. J. of Robotics Research, vol. 22, no. 7/8, pp. 479-504, 2003.

[2] A. De Luca, A. Albu-Schäffer, S. Haddadin, and G. Hirzinger, "Collision detection and safe reaction with the DLR-III lightweight robot arm," in Proc. IEEE/RSJ Int. Conf. on Intelligent Robots and Systems, 2006, pp. 1623-1630.

[3] A. De Santis, B. Siciliano, A. De Luca, and A. Bicchi, "An atlas of physical human-robot interaction," Mechanism and Machine Theory, vol. 43, no. 3, pp. 253-270, 2008.

[4] G. Hirzinger, A. Albu-Schäffer, M. Hähnle, I. Schaefer, and N. Sporer, "On a new generation of torque controlled light-weight robots," in Proc. IEEE Int. Conf. on Robotics and Automation, 2001, pp. 33563363.

[5] A. Bicchi and G. Tonietti, "Fast and soft arm tactics: Dealing with the safety-performance trade-off in robot arms design and control," IEEE Robotics and Automation Mag., vol. 11, no. 2, pp. 22-33, 2004.

[6] A. De Luca and W. Book, "Robots with flexible elements," in Springer Handbook of Robotics, B. Siciliano and O. Khatib, Eds., Springer, 2008, pp. 287-319.

[7] P. Tomei, "A simple PD controller for robots with elastic joints," IEEE Trans. on Automatic Control, vol. 36, no. 10, pp. 1208-1213, 1991.

[8] A. De Luca, B. Siciliano, and L. Zollo, "PD control with online gravity compensation for robots with elastic joints: Theory and experiments," Automatica, vol. 41, no. 10, pp. 1809-1819, 2005.

[9] A. Kugi, C. Ott, A. Albu-Schäffer, and G. Hirzinger, "On the passivitybased impedance control of flexible joint robots," IEEE Trans. on Robotics, vol. 24, no. 2, pp. $416-429,2008$.

[10] S. Haddadin, A. Albu-Schäffer, A. De Luca, and G. Hirzinger, "Collision detection and reaction: A contribution to safe physical humanrobot interaction," in Proc. IEEE/RSJ Int. Conf. on Intelligent Robots and Systems, 2008, pp. 3356-3363.

[11] M. W. Spong, "Modeling and control of elastic joint robots," ASME J. of Dynamic Systems, Measurement, and Control, vol. 109, no. 4, pp. 310-319, 1987.

[12] A. De Luca and P. Lucibello, "A general algorithm for dynamic feedback linearization of robots with elastic joints," in Proc. IEEE Int. Conf. on Robotics and Automation, 1998, pp. 504-510.

[13] A. Bicchi, S. L. Rizzini, and G. Tonietti, "Compliant design for intrinsic safety: General issue and preliminary design," in Proc. IEEE/RSJ Int. Conf. on Intelligent Robots and Systems, 2001, pp. 1864-1869.

[14] M. Zinn, O. Khatib, B. Roth, and J. K. Salisbury, "A new actuation approach for human-friendly robot design," Int. J. of Robotics Research, vol. 23, no. 4/5, pp. 379-398, 2005.

[15] S. A. Migliore, E. A. Brown, and S. P. DeWeerth, "Biologically inspired joint stiffness control," in Proc. IEEE Int. Conf. on Robotics and Automation, 2005, pp. 4508-4513.

[16] R. Schiavi, G. Grioli, S. Sen, and A. Bicchi, "VSA-II: A novel prototype of variable stiffness actuator for safe and performing robots interacting with humans," in Proc. IEEE Int. Conf. on Robotics and Automation, 2008, pp. 2171-2176.

[17] G. Palli, C. Melchiorri, and A. De Luca, "On the feedback linearization of robots with variable joint stiffness," in Proc. IEEE Int. Conf. on Robotics and Automation, 2008, pp. 1753-1759.

[18] A. De Luca, F. Flacco, A. Bicchi, and R. Schiavi, "Nonlinear decoupled motion-stiffness control and collision detection/reaction for the VSA-II variable stiffness device," in Proc. IEEE/RSJ Int. Conf. on Intelligent Robots and Systems, 2009, pp. 5487-5494.

[19] A. Isidori, Nonlinear Control Systems, 3rd ed., Springer, 1995.

[20] N. Kircanski and A. Goldenberg, "An experimental study of nonlinear stiffness, hysteresis, and friction effects in robot joints with harmonic drives and torque sensors," Int. J. of Robotics Research, vol. 16, no. 2 , pp. 214-239, 1997.

[21] T. D. Tuttle and W. P. Seering, "A nonlinear model of a harmonic drive gear transmission," IEEE Trans. on Robotics and Automation, vol. 12, no. 3, pp. 368-374, 1996.

[22] A. De Luca and F. Flacco, "Dynamic gravity cancellation and regulation control in robots with flexible transmissions: Constant, nonlinear, and variable stiffness," Università di Roma "La Sapienza", DIS Technical Report 11-2010, July 2010. 